\title{
IMBEDDING ESTIMATES INVOLVING NEW NORMS AND APPLICATIONS
}

\author{
BY MARTIN SCHECHTER
}

Let $H^{s, p}$ denote the Sobolev space with norm

$$
\|u\|_{s, p}=\left\|F\left(1+|\xi|^{2}\right)^{s / 2} F u\right\|_{p},
$$

where $F$ denotes the Fourier transform, $\bar{F}$ its inverse and $\|w\|_{p}$ is the $L^{p}\left(\mathbf{R}^{n}\right)$ norm. In many linear and nonlinear problems one comes across the question: For which functions $V(x)$ does there exist an estimate of the form

$$
\|V u\|_{q} \leq C\|u\|_{s, p}, \quad u \in H^{s, p} \text { ? }
$$

In this note we find a large class of functions $V$ for which (2) holds. To do this we introduce a new family of norms $M_{\alpha, r, t, \delta}(V)$ for $0 \leq \alpha, 0<\delta<1$, $1 \leq r<\infty$ and $1 \leq t \leq \infty$. For $x \in \mathbf{R}^{n}$ let

$$
\begin{aligned}
\omega_{\alpha}(x) & =|x|^{\alpha-n}, & & 0<\alpha<n, \\
& =1-\log |x|, & & \alpha=n, \\
& =1, & & n<\alpha .
\end{aligned}
$$

When $0<\alpha$ we define

$$
\begin{aligned}
M_{\alpha, r, t, \delta}(V) & =\left(\int\left(\int_{|x-y|<\delta}|V(x)|^{r} \omega_{\alpha}(x-y) d x\right)^{t / r} d y\right)^{1 / t}, \quad 1 \leq t<\infty, \\
& =\sup _{y}\left(\int_{|x-y|<\delta}|V(x)|^{r} \omega_{\alpha}(x-y) d x\right)^{1 / r}, \quad t=\infty .
\end{aligned}
$$

For $\alpha=0$ we put

We also set

$$
M_{0, r, t, \delta}(V)=\|V\|_{t}
$$

$$
M_{\alpha, r, t}(V)=M_{\alpha, r, t, 1}(V)
$$

and make the following basic assumption.

Hypothesis A. The parameters $\alpha, r, s, t, p, q$ satisfy:

A1. $0 \leq \alpha, s ; 1 \leq q \leq r<\infty ; 1 \leq p<\infty ; 1 \leq t \leq \infty$.

A2. $1 / q \leq 1 / p+1 / t$.

A3. $\alpha / n r \leq s / n+1 / q-1 / p-1 / t$.

A4. We do not have both $q=t$ and $n=s p$ or both $p=1$ and $s / n=$ $1 / t+1 / q^{\prime}$.

Let $M_{\alpha, r, t}$ denote the set of those functions $V(x)$ such that $M_{\alpha, r, t}(V)<\infty$.

Received by the editors July 13, 1983.

1980 Mathematics Subject Classification. Primary 47B38, 46E35; Secondary 26D10, 35J60, 45P05, 46E30, $47 \mathrm{H} 17$.

Key words and phrases. Function spaces, multiplication operators, estimates.

(C) 1984 American Mathematical Society $0273-0979 / 84 \$ 1.00+\$ .25$ per page 
TheOREM 1. Under Hypothesis (A) assume that.

(a) If $q<r$ and $s<n$, then either $p \neq 1$ or inequality A3 is strict.

(b) If $q=r$ and $s<n$, then either $r<t \leq p^{\prime}$ or inequality A3 is strict.

Let $V(x)$ be a function in $M_{\alpha, r, t}$. Then multiplication by $V$ is a bounded operator from $H^{s, p}$ to $L^{q}$. There is a constant $C_{0}$ depending only on the parameters such that

$$
\|V u\|_{q} \leq C_{0} M_{\alpha, r, t}(V)\|u\|_{s, p}, \quad u \in H^{s, p} .
$$

Moreover, there are constants $C_{1}, C_{2}$ depending only on the parameters such that

$$
\|V u\|_{q} \leq C_{1} M_{\alpha, r, t, \delta}(V)\|u\|_{s, p}+C_{2} M_{\alpha, r, t}(V)\|u\|_{p}, \quad u \in H^{s, p},
$$

and $C_{1}$ does not depend on $\delta$.

Corollary 2. If, in addition,

$$
M_{\alpha, r, t, \delta}(V) \rightarrow 0 \text { as } \delta \rightarrow 0
$$

then for every $\epsilon>0$ there is a constant $K$ such that

$$
\|V u\|_{q} \leq \epsilon\|u\|_{s, p}+K\|u\|_{p}, \quad u \in H^{s, p} .
$$

If $t \neq \infty$, then multiplication by $V$ is a compact operator from $H^{s, p}$ to $L^{q}$. The same conclusion holds when $t=\infty$ if

$$
\int_{|x-y|<1}|V(x)|^{r} \omega_{\alpha}(x-y) d x \rightarrow 0 \text { as }|y| \rightarrow \infty
$$

THEOREM 3. Let $\psi(\rho)$ be a positive function such that $\int_{0}^{1} \psi(\rho) \rho^{-1} d \rho<\infty$. Under Hypothesis A, inequalities (3) and (4) hold without the restrictions (a) and (b) of Theorem 1 provided we replace $\omega_{\alpha}(x)$ by $\omega_{\alpha}(x) \psi(x)^{-b}$ in the definition of $M_{\alpha, r, t}(V)$, where $b=r(1+1 / q-1 / p-1 / r-1 / t)$.

We apply these results to a nonlinear problem. Let $m$ be a positive integer, $\Omega$ an arbitrary (bounded or unbounded) domain $\mathbf{R}^{n}$ and let $W=H_{0}^{m, 2}(\Omega)$ be the completion of $C_{0}^{\infty}(\Omega)$ in $H^{m, 2}$. Let $a(u, v)$ be a hermitian bilinear form on $W$ satisfying

$$
K_{1}^{-2}\|u\|_{m, 2}^{2} \leq a(u)=a(u, u) \leq C^{2}\|u\|_{m, 2}^{2}, \quad u \in W .
$$

Let $f(x, v), g(x, v)$ be continuous functions on $\Omega \times \mathbf{R}$ such that $f(x, v)=$ $\partial g(x, v) / \partial v$. We assume that

$$
g(x, v) \leq B(x, v)=\sum_{k=1}^{N} V_{k}(x)|v|^{q_{k}}, \quad x \in \Omega, v \in \mathbf{R},
$$

and

$$
M_{\alpha_{k}, r_{k}, t_{k}, \delta}\left(V_{k}\right) \rightarrow 0 \text { as } \delta \rightarrow 0, \quad 1 \leq k \leq N,
$$

where

$$
1<q_{k} / 2+1 / t_{k}, \quad \alpha_{k} / n r_{k} \leq m q_{k} / n+1-q_{k} / 2-1 / t_{k} .
$$


If $t_{k}=\infty$, we assume that (7) holds for $V_{k}, \alpha_{k}, r_{k}$. By Theorem 1 there are constants $M_{k}$ such that

$$
\int_{\Omega} B(x, u(x)) d x \leq \sum_{k=1}^{N} M_{k}\|u\|_{m, 2}^{q_{k}}, \quad u \in W .
$$

Assume that

$$
K_{2}=\int_{\Omega} g(x, 0) d x
$$

exists, and put

$$
M(R)=R^{-2}\left(\sum_{1}^{N} M_{k}\left(K_{1} R\right)^{q_{k}}-K_{2}\right), \quad \lambda_{0}^{-1}=\inf _{0<R} M(R) .
$$

Let $A$ be the operator associated with $a(u, v)$ (cf. [8]), and let $\lambda>0$. We are looking for a solution of

$$
A u=\lambda f(x, u)
$$

Theorem 4. If $\lambda<\lambda_{0}$, then, for any $R$ such that $\lambda M(R)<1$, (10) has a solution satisfying $a(u) \leq R^{2}$.

There is a connection between the spaces $M_{\alpha, r, t}$ and the Lorentz spaces $L^{\sigma, t}$ (for the definitions cf. $\left.[3,11,13]\right)$.

Theorem 5. If $0 \leq 1 / \sigma-1 / t=\alpha / n r, r<\sigma \leq t<\infty$, then

$$
M_{\alpha, r, t}(V) \leq C\|V\|_{L^{\sigma, t}}, \quad V \in L^{\sigma, t} .
$$

If we combine this with Theorem 1 we obtain

TheOREM 6. If $t<\infty$ and $1 / q-1 / p \leq 1 / t \leq 1 / \sigma<1 / q, 1 / \sigma+1 / p \leq$ $s / n+1 / q$, then

$$
\|V u\|_{q} \leq C\|V\|_{L^{\sigma, t}}\|u\|_{s, p}
$$

Special cases of inequality (2) were proved by Stummel [12], Balslev [2], Berger-Schechter [4] and Schechter [7, 8, 9]. Our solution of (10) avoids some of the hypotheses of Noussair-Swanson [6]. The suggestion that there should be an inequality of the form (12) is due to $\mathrm{H}$. Brezis.

Theorem 1 is proved by using Bessel potentials as investigated by AronszajnSmith [1]. Inequality (3) is equivalent to

$$
\begin{aligned}
\left|\left(G_{s} * f, V u\right)\right| \leq & C\|f\|_{p}\|v\|_{q^{\prime}} \\
& \times\left(\int\left(\int|V(x)|^{r} G_{s}(x-y)^{r} \phi(x-y)^{-r} d x\right)^{t / r} d y\right)^{1 / t}
\end{aligned}
$$

holding for a suitably chosen function $\phi$. This is derived by tedious and tricky estimates. Corollary 2 follows by standard arguments and Theorem 3 is a slight variation. Theorem 4 is proved by variational techniques using (8) and (9). Theorem 5 is proved by using the fact that $H^{s, p} \subset L^{q}$ for certain values of $s, p, q$. By real interpolation we find that $H^{s, p} \subset L^{\sigma, p}$ for specific values. This leads to (11). Theorem 6 is merely a combination of Theorems 1 and 5 . 


\section{REFERENCES}

1. N. Aronszajn and K. T. Smith, Theory of Bessel potentials, Ann. Inst. Fourier Grenoble 11 (1961), 385-475.

2. E. Balslev, The essential spectrum of elliptic differential operators in $L^{p}\left(R_{n}\right)$, Trans. Amer. Math. Soc. 116 (1965), 193-217.

3. J. Bergh and J. Lofstrom, Interpolation spaces, Springer-Verlag, Berlin, 1976.

4. M. S. Berger and M. Schechter, Embedding theorems and quasilinear elliptic boundary value problems for unbounded domains, Trans. Amer. Math. Soc. 172 (1972), 261-278.

5. K. Jorgens and J. Weidmann, Spectral properties of Hamiltonian operators, Lecture Notes in Math., vol. 313, Springer-Verlag, Berlin, 1973.

6. E. S. Noussair and C. A. Swanson, Existence theorems for generalized Klein-Gordon equations, Bull. Amer. Math. Soc. 8 (1983), 333-336.

7. M. Schechter, On the invariance of the essential spectrum of an arbitrary operator. II, Ricerche Mat. 16 (1967), 3-26.

8. __ Spectra of partial differential operators, North-Holland, Amsterdam, 1971.

9. ___ Essential self-adjointness of the Schrödinger operator with magnetic vector potential, J. Funct. Anal. 20 (1975), 93-101.

10. B. Simon, Schrödinger semigroups, Bull. Amer. Math. Soc. 7 (1982), 447-526.

11. E. M. Stein and G. Weiss, Introduction to Fourier analysis on Euclidean spaces, Princeton Univ. Press, Princeton, N. J., 1971.

12. F. Stummel, Singuläre elliptische Differentialoperatoren in Hilbertschen Röumen, Math. Ann. 132 (1956), 150-176.

13. $\mathrm{H}$. Triebel, Interpolation theory, function spaces, differential operators, North-Holland, Amsterdam, 1978.

Courant Institute of Mathematical Sciences, New York University, New YORK, NEW YORK 10012

Current address: Department of Mathematics, University of California, Irvine, California 92717 